\title{
5 Presence and Hyperpresence: Implications for Community Awareness
}

\author{
John M. Carroll, Patrick C. Shih, Blaine Hoffman, Jing Wang, and \\ Kyungsik Han
}

\begin{abstract}
A prominent issue in community informatics is community awareness: the awareness of community members of activity in their community. Community awareness helps community members understand and appreciate their community, motivates them to participate and reciprocate, and evokes feelings of empathy, intimacy and solidarity. In this chapter we analyze community awareness technologies as helping community members feel more present to others and feel that others are more present to them. We draw upon several design investigations of supporting community awareness through aggregation of RSS feeds and Tweets, digital cultural heritage, volunteer efforts, and fieldwork understanding community awareness designs in the LiveStrong health community.
\end{abstract}

Keywords: Social Presence; Social Presence Technologies; Hyperpresence; Community Awareness.

\subsection{Introduction to Chapter 5}

Social presence is the feeling of being engaged with, and connected to others, the phenomenal sense of being together (see also Chapter 1, this volume). Conceptions of social presence derive from Goffman's $(1959,1963)$ analysis of co-presence as persons being embodied in the same place, mutually visible to and aware of one another, and socially available to one another. For Goffman, co-presence is grounded in the here-and-now physicality of the interaction situation, in one's social perception of others, and in one's awareness of being reciprocally perceived by similar others. It is the foundation for social interaction.

As a contemporary technical term, "social presence" was first used by Short, Williams \& Christie (1976) to analyze social interactions mediated by telecommunications channels. This early work emphasized the (surprising) extent to which an experience of social presence could be evoked through technologymediated human interactions, all the more surprising given the fairly low-bandwidth interactions relative to contemporary networking capabilities. Short et al. (1976) suggested further that mediated social presence is not a simple contrast of present versus absent, but rather a quality with nuances of kind and degree, potentially modulated by relationships among participants, by the salience of a given participant

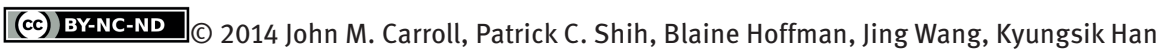
This work is licensed under the Creative Commons Attribution-NonCommercial-NoDerivs 3.0 License. 
in a given interaction, as well as by attitudes towards the technological mediation, in their case, the telecommunications channel.

Contemporary networking infrastructures enable a great variety of what Biocca, Harms and Burgoon (2003) call social presence technologies, giving high-bandwidth teleconferencing, collaborative work environments, mobile and wireless interactions, speech interfaces, and 3D social virtual environments as examples. Various social presence technologies enable a wide range of social presence phenomena, such as the experience of remote interaction mediated by avatars (Bailenson et al. 2008). Biocca et al. also emphasize that we do not have a comprehensive theory of social presence, as they describe it, "of how differences in technological connection, representation, and mediated access affect, distort, or enhance the perception (mental model) of others' intentional, cognitive, and affective states and behavior resulting from those perceptions.” (Biocca et al., 2003, p. 473).

In this chapter, we focus on place-based and community technologies that amplify sense of presence in the embodied world, and on particular ways that such technologies may amplified social presence. In order to assess and analyze the role and effects of social presence in any situation, we must understand how technologymediated interaction reveals important characteristics that shape and influence the perception of social presence. We report a design research investigation of social presence for interactions among persons living in a placed community. Community members are often co-present, in Goffman's sense, as they move about in local public space. However, their interactions can also be mediated, through interactions with local information and services. We coin the term hyperpresence to refer to social presence heightened beyond what is (normally) possible face to face, on analogy to the term hyperlocal news, news about a locale created by people at or in that locale for the use of people at or in that locale (Farhi, 1991).

We illustrate hyperpresence through several design inquiries. CiVicinity is a platform that utilizes community feeds such as news, events, and discussions to make people more aware and feel more present in their community. Smartphone applications such as Lost State College, Local News Chatter, and Mobile Timebanking add the dimension of mobility to hyperpresence, and allow community members to more easily contribute to a continuing discourse of community topics. We describe our studies of an online virtual community: LiveStrong.com, and how various designs afford the sense of social presence in a virtual community. In each of these, the capabilities of the technology support feelings of hyperpresence that would not arise otherwise.

\subsection{Hyperpresence in Local Communities}

In our work with community partners and groups, our research group has established a community-driven portal site for the surrounding area. Typically, the information 
generated by and within a given community originates from a number of sparse sources, each generating seemingly little information over time. Many of these sources might employ feeds (e.g., RSS, ATOM, iCalendar), a technology that enables updates and information to be automatically pushed to subscribers, as opposed to requiring readers to discover and check a web site manually.

Built upon the foundation of previous prototypes, CiVicinity is a web-based community portal that employs Web 2.0 assets to deliver up-to-date community information and news closely associated with community places and members. CiVicinity populates its news page and its events calendar with data from communityowned feeds. It also incorporates location-sensitive, event-based features and expands upon the idea to pair maps and coordinate data across the whole site. The site serves as a centralized aggregate of community information, reducing the effort required for any individual to access and peruse the information. Each news feed entry on CiVicinity provides a link to the original source, enabling readers to easily navigate to the blog, news site, organization's web page, and so on. Categories identify the type of feed source, making it easier for readers to seek specific types of stories and to filter the feeds by interest. On the events calendar page, we parse iCalendar feeds from community sources to list events in a calendar view. As with the news feeds, the description of these events includes the name of the source organization. Since news and calendar feeds sometimes provide geocode data, we pair news and calendar information with a map visualization, provide a view that sorts lists and items by distance from the user, and provide directions to events and addresses. CiVinicity is integrated with social networking sites such as Facebook, Twitter, and email using the respective APIs provided by these platforms. Any news story or event that catches a reader's interest can be shared. Our prior prototypes were deployed in support of specific meta-events, such as a local arts festival and holiday activities on New Year's Eve, focusing on various activities and locations within the community.

Using CiVicinity, individuals can all be on the same page about the relevant where of information. For example, the explicit use of map visualizations enhances awareness of community events and their relevance to planning social interactions and shaping an understanding of the community itself (Hoffman et al, 2012). The aggregated community information portal creates a virtual space that augments the physical spaces of the community to create a more complete community place. As a result, CiVicinity is part of the process of creating and maintaining social presence rather than a separate arena of presence itself. Furthermore, the use of CiVicinity can convey presence regardless of synchronous use or concurrent physical co-location. The commenting and interactions associated on the site, such as reading historical information about a place while also visiting that place or viewing the comments left by others about community news and events, help to affirm the feeling of others' presence within the community. Exploring community places and communitygenerated discussion is not reliant upon individuals meeting up in person. CiVicinity's online existence and digital nature afford interactions not possible in face-to-face 
Calendar Help Submit an Event!

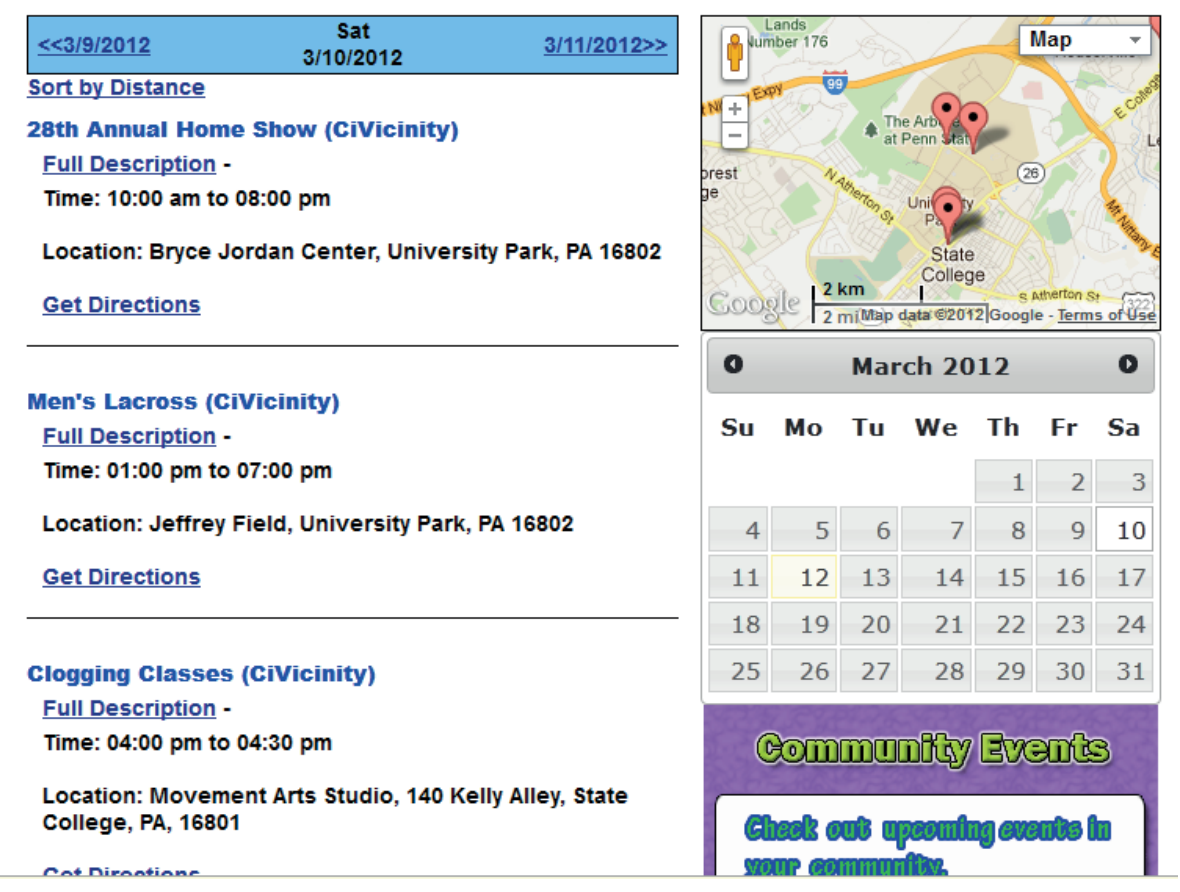

Figure 5.1: Screenshot of the CiVicinity Events page

settings. The timing of an individual's travels throughout town no longer dictate whom he or she might bump into, just as strangers in the same crowd that might never interact could find themselves engaged with others using the site. CiVicinity makes individuals and events more available to the community.

With CiVicinity, individuals are more present in their community due to removing restrictions of space and time. Civicinity presents a more complete and clear overview of the community via maps that show where interactions are, or will be, and enables community members to share in knowing who is involved. CiVicinity is meant to raise community awareness among community members. By highlighting what is being discussed and what is happening within a community and delivering that information directly to individuals, their overall understanding of the community increases. Being accessible at any time and explicitly keeping location and position at the forefront of interactions increases the understanding of technology's ability to enhance and supplement activity. Together, these two characteristics foster an easier acceptance and comfort of the frame of interaction across the community via technology. Technologymediated interaction that aggregates community information, geolocation, and social media is embraced by CiVicinity to enhance the construction of hyperpresence. 


\subsection{Hyperpresence in Mobile Technologies}

Expanding on enhancing hyperpresence in the same geographical communities with traditional Web 2.0 technologies, our research group has been investigating how mobile platforms would make people more aware of and ease participation in various local community news, events, or activities. Although a great amount of concern with social isolation due to the Internet and mobile device does exist, many recent studies have indicated that technology use contributes to having more social connection, interaction, and participation (Hampton et. al, 2009). Similarly, by analyzing national survey data of internet usage and friendship, Wang and Wellman demonstrate that technologies blurred boundaries between online and offline and people who use the Internet and mobile technology still interact with others on a regular basis and the number of friends itself even increased because of the popularity and penetration of social media (Wang \& Wellman, 2010).

A growing number of people adopt and use mobile devices in their daily lives and start to leverage its unique opportunities, such as personalization, portability and ubiquitous connectivity, to facilitate and reinforce their social interactions and connections with other people (Carroll \& Rosson, 2009). Local residents' presence within a geographical community can be mediated by the use of their mobile devices, which would lead to the construction of a social presence in which one's activities and actions become more visible to their community as well as other community members. To articulate the notion of hyperpresence and social interaction mediated by mobile technology in a local community context, we have been extensively working on three projects that leverage the capabilities of smartphones to support local heritage (Lost State College), local news and events (Local News Chatter), and local volunteer efforts (Mobile Timebanking). Each project has a designated smartphone app that enables enhanced community awareness, participation, and engagement and social connection and interaction, and thereby one's sense of social presence.

Lost State College (LSC) is a smartphone app to make local cultural heritage more visible and available to community members, aiming to capture and share the collective memories of a town's history (Han et al., 2014). The meaning of LSC implies preservation efforts of local history; otherwise it will be "lost" in the future. LSC utilizes a geo-coded dataset of a number of historic downtown landmarks provided by a Borough of State College. For each location, LSC provides a name, address, a set of official historical and current photos, a text description, and a pre-recorded audio description for each landmark. Along with these official content, LSC also provides four additional interfaces (named Social Features) for social interactions in which people can add their personal reflections on and stories to the landmarks by augmenting visits, likes, comments, or photos on their mobile device.

Our user study with local residents indicates that participants both utilized official and social information when appreciating the landmarks through LSC. In particular, by adding their own or accessing others' photos, comments, likes, and visits, social 


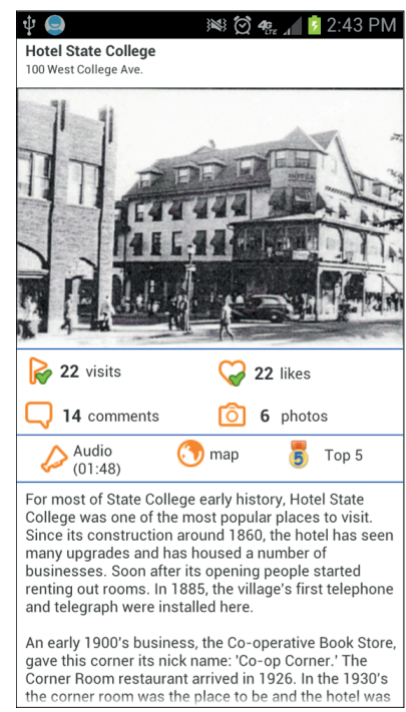

(a)

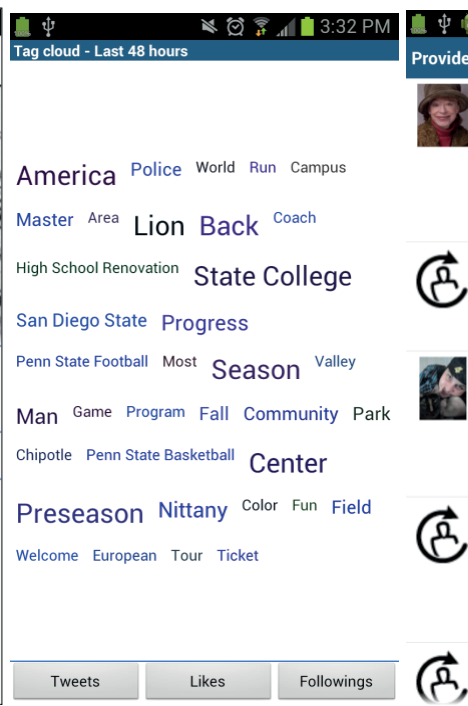

(b)

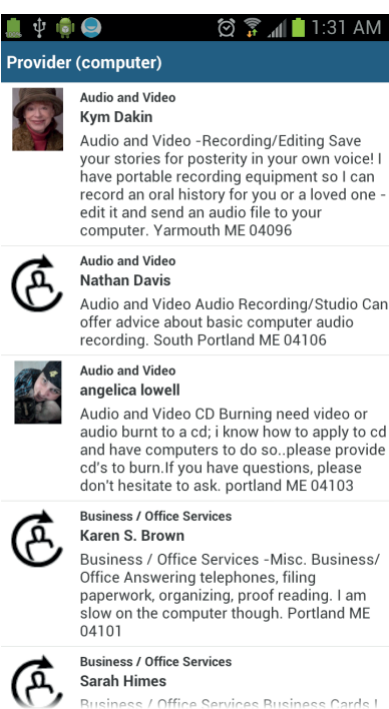

(c)

Figure 5.2: Screenshots of (a) Lost State College, (b) Local News Chatter, and (c) Mobile Timebanking

features allowed participants to not only increase their awareness of local landmarks and history but also reconstruct landmarks into more dynamic and socially meaningful places to themselves and their community. LSC enabled participants to realize that the place where they live has its own long and rich history as well as giving them heartfelt appreciation for the people and families who built up the town over many generations. Some participants mentioned,

“I really didn’t know buildings have some stories and history. Giving more different perspectives to my community" (Han et al., 2014: Participant 20) and "I have learned a lot about this community, like it used to be a bank, theatre, and it got burned, etc. I think it is a learning experience" (Han et al., 2014: Participant 28).

They also mentioned that they felt more identity and belonging to a community after completing the tour.

"I love to know about the story of each building. Knowing a little bit more makes me feel even more like 'townie'” (Han et al., 2014: Participant 30).

Using and interacting with the app suggests that local residents' use of mobile technology evoked different perspectives and feelings of social presence not present otherwise. Local landmarks that merely existed as part of the environment and subconsciously acknowledged as people walked by every day became focal points of interaction and historical meaning. 
Local News Chatter (LNC) is the local new aggregator that integrates sociallygenerated local tweets and formal local news feeds to elicit new and unique community value (Han et al., 2013). Users would see local stories along with local tweets relevant to those stories. Since local news and tweets are publicly available data, no additional effort from the users is required in creating this content. Rather, existing content is leveraged through keyword analysis that organizes stories with relevant tweets. When users see the tweets about stories that are interesting or relevant to their lives, they can follow up with the other community members who made the tweets. As a result, community members benefit from better community awareness of local issues and personal perspectives and can share their interests and concerns without any content creation cost. LNC utilizes relevant local topics reported in the newspapers and existing Twitter comments of local residents about these topics to provide a local discussion space. LNC makes its users more aware of the community knowledge and sentiment about local topics and enhances the social presence of other members in the community who will otherwise remain invisible to the users, thereby achieving hyperpresence.

Based on the user study with local residents, we found that participants appreciated accessing a rich amount of local news information from the formal news and the social media. They in particular liked reading tweets from other community members, because it broadens their insights on local community topics.

"Pointed me to news stories or insights about the local area which I might not know (and therefore be interested in) otherwise" (Han et al., 2014: Participant 22) and "Yes, I enjoyed the festival tremendously. It's good to realize that almost everyone feels the same way" (Han et al., 2014: Participant 18).

This also affected their participation and engagement in local news information.

"I could see people's interests and eager for support the apartment residents [who suffered from a fire]. People retweeted the way how to donate, the motivation of fire, and so forth. [...] I was very interested in how to help and what I can do to help them” (Han et al., 2014: Participant 29).

Many participants expressed that they wanted to engage more in news activities by creating personal thoughts or opinions, retweeting existing ones, or following other users to receive their updated news in the future.

Lastly, Mobile Timebanking is the extension of conventional timebanking, which uses time as a form of currency to encourage service exchanges among timebank members in the same community (Bellotti et al., 2013; Carroll, 2013). For example, one resident might be a competent handyman, while another plays guitar. Each can provide community service doing what he or she can do for other members and receives time credits that can be exchanged for services from other members, such as gardening. Since timebanking requires interpersonal communications and interactions, much research on timebanking has shown that it strengthens interpersonal trust, social connection 
and the sense of community attachment (Collom et al., 2012). With this goal in mind, our design research of leveraging mobile technology in a timebank context attempts to take advantages of the affordances of smartphones, such as facilitating access to communications and transaction management activities. The Mobile Timebanking app supports not only the basic timebanking functionalities of posting, accessing tasks, giving time credits, and accessing task history and user's profile details but also those that are suited to the use of smartphones, such as having a map view that shows both user and task locations and notifying users of any incoming text messages from other users or status updates of the tasks in near-real time.

Our user study with local residents reveals how hyperpresence of local volunteer efforts is mediated by mobile technology. We found that participants were surprised by the fact that there was a lot of tasks posted by others and were pleased to discover that many people were willing to provide help in the community. The local volunteer interactions and activities was made more visible to other people, the local volunteers were made more accessible by the mobile platform because their offers were easily shared and accessed by their mobile device, and people were able to respond to urgent requests more easily with the app. Mobile Timebanking shows a potential of fostering community altruistic exchanges and creating social connections by using one's skills or resources to help others.

Overall, mobile computing enables the use of hyperlocal information, wherein users' location can be capitalized upon and used to tailor the collection and delivery of community knowledge and social communication. Our investigations through Lost State College, Local News Chatter, and Mobile Timebanking show that information delivered over mobile platforms can enhance the experience in-situ, extending the interaction beyond the tangible and crossing spans of time to connect individuals. As a result, the technology transcends a role of connecting remote or disparate individuals to serve as a platform that extends the notion of place beyond the physical spaces upon which a community is built, and generates a notion of hyperpresence in the community not tied to physical interaction alone.

\subsection{Hyperpresence in Online Virtual Communities}

As Web 2.0, social media, and other communication technologies become more widely adopted, more and more people are collaborating, communicating, and meeting with friends and even strangers in a purely virtual environment with no physical face-toface interactions. In order to understand how social presence takes place in virtual communities with no physical, face-to-face interactions, we conducted an empirical study of LiveStrong.com, an online health community, and how technological affordances help enable social presence in a virtual community platform.

LiveStrong.com is a platform that supports several health conditions but is primarily known for its online weight management community and its calorie- 
tracking tool with data sharing capability. It was launched in 2008 and had over four million registered users as of 2011. The community supports member interactions in its blog-style groups. Members can create custom groups that are either open to public or private to group members. Many active and popular groups have thousands of members. For example, the 100+ Pounds to Lose Group has more than 4,000 members. Group spaces are organized in a blog format: they are segmented by dates with members' posts and corresponding comments constituting each segment. Anyone can post open-texts to a public group without membership; by joining a group, one's tracking data will be aggregated with the data of other group members. The MyPlate calorie tracker allows users to log their food and fitness activities manually. Users can select their daily or weekly weight management goal to be losing, gaining, or maintaining a specific amount of weight. MyPlate also enables users to customize their ultimate weight and daily nutrient goals. Weight progress is visualized in a line chart as well as in a highlighted note, such as, "I've lost <amount of pounds> lbs!". Individual users' daily tracking data of calorie goals, calories consumed, logs of food intake and calories of each food item and its nutrient breakdown, calories burned, logs of fitness activities, and net calories consumed can be presented and shared in two ways. One is called Food Diary, an integral record that consists of tracked food and fitness information and allows comments from other members. Users can configure which items of the tracked data are to be shown in the diaries and who can access their diaries (i.e., public, friends only, private, and selected individuals). The other is an aggregate view of group members' daily tracked data, which summarizes parts of the individuals' data. Both total and average values of these measurements from members who have tracked their activities on that day are shown in a table, which can also be expanded to display data at the individual level. Groups can choose whether to enable this visualization feature in their group space.

Presence of LiveStrong.com users is mediated by both the community spaces and the tracking technology. As the core benefit of online health communities, the presence-the sense of availability-of a large support network was cited as the one of the critical criteria participants employed to decide which LiveStrong.com group to join. Such presence is mainly indicated by regular posting from group members. In contrast to face-to-face support groups, these online groups do not emphasize real-time responses to people's frustration, anxiety, or concerns. Moreover, people do not expect support from a specific person, accepting it from anyone as long as it is positive and constructive. What matters is the sense that someone will be there within a certain period of time when an individual needs support. Every member benefits from others' presence; at the same time, their presence contributes to other members' weight management processes. The following example illustrates how participating in LiveStrong.com with others who share similar experiences generates a sense of presence among the community members. 
"I like this group because I feel there is a fellowship amongst us who have lost a lot of weight or who are in the process of it. There is nothing better than having someone who has been there along for the ride" (Wang et al., 2014: Participant 9).

Aside from creating the perceived social presence of a large support network, LiveStrong.com, like other online health communities, also mediates such presence by grouping people with similar experiences and attributes. Different from physically presenting oneself, which is often enabled by co-location or rich media (e.g., video, virtual modeling), LiveStrong.com heightens a dimension of individual identity, making it salient and easy to relate to for people. The majority of participants mentioned the importance of such similarities: peers sharing similar health-related experiences were especially competent to offer empathy and pertinent informational support, keeping them engaged in the community.

“I initially joined the 5'2" group because there are constraints for a short person working out that tall people don't get. However, I quickly veered away from this group because while the group members meet the 5'2" or less criteria, this community is comprised mostly of women in their 20's who have no idea what it's like to be short and old and trying to get and stay fit and healthy. 8-)... The [Getting Fit and Fabulous Over 40] group is not constrained by a specific height, but they are all people (primarily women) in their 40's or thereabouts who share their experiences..." (Wang et al., 2014: Participant 1).

The tracking data generated by individuals, either narratives (e.g., amount of weight loss per week shared in group discussions) or structured daily activities (e.g., food diaries), create opportunities of new ways of self-presentation in the community. It is hardly possible for peers to maintain awareness of one's every food intake and exercise. It is also difficult for them to generate a holistic view of one's overall progress towards his/her calorie goals as well as weight goals. The tracking tool enables all the information to be captured and accessed at various levels of detail beyond individual's own cognitive capabilities. This provides the basis for peers to exchange social support with each other.

"I met all of these 'friends' in various groups that I was active in before. We can 'visit' each others diaries, read what they wrote and make comments. We often:

---Encourage a friend if they're down.

---Compliment them on having a nice "plate" of tracked food.

---Comment specifically on what they wrote and the things they said.

---Ask questions about what they wrote, or the food they tracked.

In my 'circle', there are between 3-10 ladies who "run around" visiting each other's diaries and posting nice comments. If someone posts a comment to your diary, it's only courteous to return the favor and share the love. It creates a kind of reciprocal community" (Wang et al., 2014: Participant 3).

At LiveStrong.com, aggregating group tracking data of calorie goals and intake was intended to create interdependence and accountability among group members; 
however, such a design failed to achieve this purpose because many users did not regularly log into the system or completely recorded their activities. Instead, participants found the mutual awareness of weight loss progress-the amount of weight lost-during weekly weigh-in activities effective in sustaining their motivations to achieve their goals.

"I fed on the Monday weigh-ins and Tuesday summaries, and took great delight as I progressed up the 'weight list' ladder” (Wang et al., 2014: Participant 7).

Constraining one's presence by not disclosing one's own tracking data, on the contrary, was perceived as detrimental to goal achievement.

"I notice that those who complain the most about not being able to get results often don't make their food diaries public and that leads me to believe that they're not honest with themselves or others. If you're eating right and exercising enough, you WILL lose the weight!” (Wang et al., 2014: Participant 4).

Although members in a virtual community encounter certain challenges that do not apply to geographical communities, such as sharing a common reference of tangible objects that exhibited prior shared understandings, technologies such as activity trackers and awareness feeds allow members to receive social support and be upto-date about other members' progress. Hyperpresence is enhanced even without physical and face-to-face interactions.

\subsection{Conclusion to Chapter 5}

Social presence is the quality and degree of mutual visibility and social availability (Goffman, 1959; 1963; Short et al. 1976). It can be evoked in mediated interactions even when they are relatively brief and instrumental (telecommunications, remote collaborative work, e-commerce and online help, and 3D social virtual environments; Biocca et al., 2003).

We analyzed social presence in community contexts that involve technology mediation; we argued that community awareness technologies help community members feel more present to others, and feel that others are more present to them, than they might feel interacting only face-to-face. These technologies can help community members to be aware of more (that is, a greater number and diversity of community news, events, places, opinions), but also more aware (that is, more deeply aware of what others are doing and achieving, what they care about and tweet about, how they manage challenges). We coined the term hyperpresence to describe such situations.

Social presence is important to communities. High mutual visibility and social availability enable the key beliefs, behaviors, and emotions that constitute community membership, identity and belonging, participation and reciprocity, empathy, 
intimacy and support. Contemporary community is in some jeopardy (Putnam, 2000). Supporting hyperpresence more effectively could be an important direction for community informatics.

\section{References}

Bailenson, J. N., Yee, N., Blascovich, J., \& Guadagno, R. E. (2008). Transformed social interaction in mediated interpersonal communication. In E. Konijn, M. Tanis, S. Utz \& A. Linden (Eds.), Mediated interpersonal communication, Mahwah, NJ: Lawrence Erlbaum Associates, 77-99.

Bellotti, V., Carroll, J. M., \& Han, K. (2013). Random acts of kindness: The intelligent and contextaware future of reciprocal altruism and community collaboration. Proceedings of IEEE CTS 2013: International Conference on Collaboration Technologies and Systems (San Diego, CA, May 20-24). IEEE, 1-12.

Biocca, F., Harms, C., \& Burgoon, J. K. (2003). Towards a more robust theory and measure of social presence: Review and suggested criteria. Presence: Teleoperators and Virtual Environments, 12 (5), 456-480.

Carroll, J.M. (2013). Co-production scenarios for mobile time banking. In Y. Dittrich et al. (Eds.). Proceedings of IS-EUD 2013: Fourth International Symposium on End-User Development (Copenhagen, Denmark, June 10-13). Lecture Notes in Computer Science, LNCS 7897. Heidelberg: Springer, 137-152.

Carroll, J. M. \& Rosson, M. B. (2009). Theorizing mobility in community contexts. Journal of Human Computer Studies, 66(12), 944-962.

Collom, E. Lasker, J., \& Kyriacou, C. (2012). Equal Time, Equal Value: Community Currencies and Time Banking in the US. Ashgate Publishing, Ltd.

Farhi, P. (1991, March 11). Taking Local Coverage to the Limit: 24-Hour Cable News, Washington Post, Financial Section, 25.

Goffman, E. (1959). The presentation of self in everyday life. Garden City, NY: Anchor.

Goffman, E. (1963). Behavior in public places: Notes on the social organization of gatherings. New York: The Free Press.

Hampton, K., Sessions, L., Her, E. J., \& Rainie, L. (2009, November). Social Isolation and New Technology: How the internet and mobile phones impact Americans' social networks, Pew Internet Project.

Han, K., Shih, P. C., \& Carroll, J. M. (2013). Aggregating community information to explore social connections. When The City Meets The Citizen Workshop, ICWSM 2013: 7th International AAAI Conference On Weblogs And Social Media (July 8-11, Boston MA).

Han, K., Shih, P. C., Rosson, M. B., \& Carroll, J. M. (2014). Enhancing Community Awareness of and Participation in Local Heritage with a Mobile Application. Proceedings of the 17th ACM Conference on Computer Supported Cooperative Work \& Social Computing (Baltimore, MD, Feb 15-19). New York, ACM Press, 2014.

Hoffman, B., Robinson, H., Han, K., \& Carroll, J. M. (2012). “CiVicinity events: pairing geolocation tools with a community calendar". Proceedings of the 3rd International Conference on Computing for Geospatial Research and Applications (COM.Geo '12). ACM, New York, NY, USA.

Putnam, R. (2000). Bowling Alone: The Collapse and Revival of American Community, New York: Simon \& Schuster.

Short, J., Williams, E., \& Christie, B. (1976). The social psychology of telecommunications. London: John Wiley and Sons. 
Wang, J., Shih, P. C., and Carroll, J. M. (2014). Life after Weight Loss: Challenges and Opportunities in Designing for Weight Management Communities. Manuscript submitted for publication.

Wang, H. \& Wellman, B. (2010). Social Connectivity in America: Changes in Adult Friendship Network Size From 2002 to 2007. Journal of American Behavioral Scientist, 53 (8), 1148-1169. 